\title{
P02-1-74 Poster session
}

\section{Protective effect of the general anesthetic: Propofol on the intracranial hemorrhage}

\author{
Xiao-Chun Zhao', Ping An ${ }^{2}$

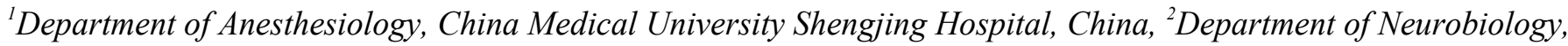 \\ China Medical University, China
}

Background:As the population ages, patients with a history of stroke will increasingly require surgery or sedation. Intracranial hemorrhage is the most lethal type of central system disease. It is important to be able to provide safe anesthesia with neuroprotective potential in such cases. Propofol is a kind of general intravenous anesthetic widely used in many kinds of surgery and has been the first choice to sedate the brain trauma patients. Little is known about the mechanism of propofol in mice with intracerebral hemorrhagic stroke injury. In this study, we examined the brain injury volume, neurologic deficit, the level of ROS, and the NADPHase activity in mice treated with propofol or vehicle after collagenase-induced intracerebral hemorrhage (ICH). Methods: ICH was induced in 12-month-old C57BL/6 mice by intrastriatal injection of collagenase. Propofol $(20 \mathrm{mg} / \mathrm{kg} / \mathrm{h})$ or vehicle $(10 \%$ intralipid $)$ was injected intravenously $2 \mathrm{~h}$ after collagenase injection for $4 \mathrm{~h}$. Results: The brain injury volume on day 3 after ICH was reduced in the Propofoltreated groups compared to that in the vehicle-treated control groups $(\mathrm{P}<0.01)$. Similarly, neurologic deficit scores were lower in mice treated with propofol than in those treated with vehicle on day 1 and on day 3 after ICH ( $\mathrm{n}=15 /$ group, all $\mathrm{P}<0.01$ ). In the hemorrhagic brain, the level of ROS was measured by In situ detection of oxidized hydroethidine. Oxidized hydroethidine fluorescence surrounding the hematoma in propofol treated mice was significantly reduced than that in the vehicle control mice at 24 hours after ICH throughout all levels of the brain $(\mathrm{P}<0.01)$. NADPHase activity around hematoma were also reduced in the propofol treated mice group than that in the vehicle control group 24 hours after ICH $(\mathrm{P}<0.01)$. Conclusion: Our results indicate that propofol significantly reduces the expansion of brain injury and neurological deficits after ICH. This protection may due to the inhibition of activated NADPHase activity. 